\title{
Autonomie für die Dritte Gewalt - Erwartungen aus der Politik
}

\section{Einleitung}

Der Deutsche Richterbund hat auf der Bundesvertreterversammlung im April 2007 die Selbstverwaltung der Justiz zu einer zentralen Verbandsforderung erhoben. Die größte berufsständische Vereinigung der Richterinnen und Richter hat sich damit an die Speerspitze einer Bewegung gesetzt, die auf umfangreiche Vorarbeiten innerhalb dieses Berufsverbandes und der weiteren Richterverbände sowie auf intensive Auseinandersetzungen mit der Thematik in der Wissenschaft zurückgreifen kann. Die Debatte um die Selbstverwaltung der Justiz hat seitdem die Bereiche der Berufsverbände und der Wissenschaft zwar nicht verlassen - wie man heute auf dieser Veranstaltung eindrucksvoll sieht. Sie findet aber nunmehr zusätzlich auch auf einer weiteren Ebene statt: In der öffentlichen Auseinandersetzung und vor allem in der Politik. In Hamburg hat die schwarz-grüne Landesregierung die Forderung nach einem »ergebnisoffenen Diskussionsprozess « zur Einführung der Selbstverwaltung der Justiz nunmehr erstmals auf die politische Agenda eines Bundeslandes gesetzt. Auch aus Schleswig-Holstein hört man neue Töne. Der dortige Justizminister Uwe Döring hält in öffentlichen Erklärungen gar seine eigene Abschaffung für eine mögliche Folge der Einführung einer Selbstverwaltung der Justiz und will diesen Prozess zügig zum Abschluss bringen.

Was treibt die Politik um, dieses Thema auf die Tagesordnung zu setzen? Was erwartet sie von einer Stärkung der Autonomie der Justiz? Wer profitiert von ihrer Einführung?

\section{Erwartung 1: Qualität und Unabhängigkeit des gerichtlichen Personals profitieren bei der Selbständigkeit der Personalauswahl von der Exekutive}

Einer der Schwerpunkte jedes Modells zu diesem Thema ist die Auswahl des Personals der Justiz im Eingangs- und Beförderungsamt. Schon bald nach Inkrafttreten des Grundgesetzes und danach immer wieder wurde der zu hohe Einfluss der Exekutive auf die Personalauswahl beklagt. Sind die Justizverwaltungen auch - aus guten Gründen - gehindert, direkten Einfluss auf die Entscheidungen der Gerichte auszuüben, so wäre es doch - wie Ihnen allen bekannt ist - eine Illusion, nach gegenwärtiger Praxis im Bereich der Personalauswahl eine Sphäre völliger Machtentsagung der Exekutive $\mathrm{zu}$ vermuten.

Wir sollten eine Struktur für die Personalauswahl der Gerichte entwickeln, die dem Prinzip der Bestenauslese wieder stärkeres Gewicht verleiht und den Machterhaltungsanspruch der Exekutive demgegenüber zurückstehen lässt. Aktuelle Vorgänge zeigen, dass sich dahinter eine Problematik verbirgt, die nicht nur für Verfassungsspezialisten interessant sein dürfte. In Italien wird von Seiten der Regierung massiv versucht, über Gesetzgebungsverfahren auf die inhaltliche Entscheidung von Gerichten Einfluss auszuüben. Stellen wir uns in Deutschland eine Regierung mit dieser Agenda vor, verbunden 
vielleicht mit Einfluss auf Teile der Medienlandschaft. Dann aber auf der anderen Seite ohne die tief verankerte Selbstverwaltung der Justiz in Italien, von der auf diesem Seminar noch die Rede sein wird, sondern mit all den Einflussmöglichkeiten auf die Personalauswahl, die heute Justizministern in Deutschland zur Verfügung stehen. Es würde sich schnell zeigen, dass die Bedrohung für die Unabhängigkeit der Rechtssprechung kein rein akademisches Problem mehr wäre. Deswegen gilt es, die Sicherung dieser Unabhängigkeit der Rechtsprechung schon heute nach Kräften krisenfest zu machen.

Im Vordergrund der Personalentscheidungen vom kleinsten Amtsgericht bis hin zu den Bundes- und Landesverfassungsgerichten muss die Qualifikation der Richterinnen und Richter für die ihnen anvertraute Tätigkeit stehen. Zugleich bedarf die mit dem Richterberuf auf Lebenszeit verliehene Möglichkeit und Pflicht zur unmittelbaren Ausübung von Staatsgewalt der demokratischen Legitimation. Das Demokratieprinzip stellt für jede Überlegung einer vollständigen Selbstrekrutierung der Justiz zu Recht eine unüberwindbare Hürde dar. Es darf auch in der Justiz keine Legitimationslücke bei der Ausübung staatlicher Gewalt entstehen. Mithin wird es unabdingbar sein, ein Wahlgremium zu schaffen, dem auch Mitglieder angehören, die durch demokratische Wahlen legitimiert wurden. Ein solches Gremium für die Richterwahl wird damit niemals zu einem völlig politikfreien Raum werden können. Es sollten aber ggf. neben anderen Professionen, etwa aus der der Rechtsanwaltschaft, Menschen hinzutreten, die aus der Mitte der Richterinnen und Richter gewählt werden.

Ob ein solches Wahlgremium dann am Ende mehr Ähnlichkeit mit dem vom Deutschen Richterbund vorgeschlagenen Justizverwaltungsrat hat, oder sich eher an die heute bereits existierenden Richterwahlausschüsse anlehnt, ist aus meiner Sicht nicht die entscheidende Frage. Wichtig ist, dass das Wahlgremium demokratisch legitimiert und pluralistisch zusammengesetzt ist, um Auswahlentscheidungen zu treffen, die noch stärker als dies heute der Fall ist, die Unabhängigkeit und Qualität der Rechtsprechung gewährleisten.

\section{Erwartung 2: Die Bürgerinnen und Bürger profitieren von einer weiteren Steigerung der Effizienz der Dritten Gewalt}

Die Gerichte in Deutschland stehen heute unter einem erheblichen Spardruck. Einem Zuwachs an Aufgaben und einer Vermehrung von Prüfungs- und Aufklärungspflichten steht im besten Fall eine gleich bleibende Stärke des Personalkörpers gegenüber. Manchmal nicht einmal das.

Eine Reaktion hierauf bestand darin, die »Neuen Steuerungsmodelle« auf die Justiz zu übertragen. Vereinfacht gesagt geht es hierbei um Modelle, welche die Leistungen der Justiz in stärkerem Maße messbar und vergleichbar machen sollen, sicherlich auch mit dem Hintergedanken aufgrund der gewonnen Ergebnisse Effizienzgewinne zu erschließen. Die Versuchung der Politik, bewährte Modelle aus der Betriebswirtschaft und der Verwaltungswissenschaft auf die Justiz zu übertragen, ist in Zeiten der schwierigen Haushaltspolitik aus meiner Sicht verständlich. Ebenso verständlich aber sind Widerstände aus der Justiz, die ihren Grund in der von den politischen Entscheidungsträgern nicht immer in ihrer vollen Tragweite erfassten Unabhängigkeit der Rechtsprechung haben. 
Es macht, davon bin ich überzeugt, den entscheidenden Unterschied, ob Modelle der Effizienzsteigerung in den Amtsstuben des Justizministers konzipiert und von dort aus kontrolliert werden. Oder ob die in den Gerichten Beschäftigten selbst eigenverantwortlich nach Wegen suchen, mit den knappen Ressourcen verantwortungsvoll umzugehen. Es ist wahr: Auch bei Einführung einer Autonomie der Justiz findet die Selbstverwaltung und damit auch die Effizienzsteigerung vor der Unabhängigkeit der Rechtsprechung ihre Schranke. Die selbstverwaltete Justiz kann aber erstens die Unterscheidung zwischen dem Bereich der unabhängigen Rechtsprechung einerseits und der dem Effizienzgedanken zugänglichen Verwaltungstätigkeit der Gerichte andererseits zielgenauer treffen. Sie kann zweitens darauf bauen, dass die Gerichte den verantwortungsvollen Umgang mit den finanziellen Ressourcen als eigene Angelegenheit ansehen, und nicht als Vorgabe einer Landesregierung, die man nolens volens umzusetzen hat. Dies gilt umso stärker, wenn es uns gelingt, der Justiz die Effizienzgewinne zur eigenen Verwendung zu belassen. Die Dritte Gewalt kann damit den Ansprüchen der Bürgerinnen und Bürger an eine leistungsfähige Justiz auch bei zunehmenden Aufgaben angemessen genügen.

Es tritt hinzu der allgemeine Grundsatz der Subsidiarität. Das Wissen um die Grundlagen der zu treffenden Entscheidungen nimmt gewöhnlich mit zunehmendem Abstand von den Orten ihrer Auswirkung ab. Dies führt zum Vorrang des Prinzips der »Entscheidung vor Ort«. Es hat sich in vielen Bereichen der Verwaltung durchgesetzt, etwa im Forschungs- und Bildungssektor, wo seit Jahren der Trend hingeht - zu mehr Autonomie. Es würde mir nicht einleuchten, weshalb ausgerechnet in der Justiz die zentrale Steuerung der Mittelverwendung der Entscheidung selbständiger dezentraler Einheiten überlegen sein sollte. Erfahrungen, die wir nicht nur in Hamburg im Rahmen des Projekts Justiz 2000 sammeln durften, sondern die inzwischen auch in anderen Projektgerichten mit Budgetverantwortlichkeit bestätigt wurden, sind unter dem Strich positiv.

Diese Erwägungen gelten im Grundsatz ebenso für die Staatsanwaltschaften. Auch die Arbeit der Staatsanwältinnen und Staatsanwälte würde nach meiner Einschätzung von einer Einführung der Selbstverwaltung profitieren. Sie ließe sich wegen der bereits jetzt bestehenden gemeinsamen Organisationsstrukturen und des in der Natur der Sache liegenden fachlichen Austauschs auch einbetten in ein Gesamtkonstrukt »Selbstverwaltung für die Justiz«. Wir dürfen allerdings eins nicht aus dem Auge verlieren: In Hamburg haben wir im Anschluss an einen sog. Ehrenmord im ersten Halbjahr des Jahres 2008 die Kommunikationsprozesse zwischen den staatlichen Behörden sehr genau unter die Lupe genommen. Auch wenn wir bei dieser Aufarbeitung diesmal kein sog. Behördenversagen feststellen konnten, sind wir natürlich auf Möglichkeiten gestoßen, die Prozesse weiter zu verbessern. Die Staatsanwaltschaft, die einen noch unmittelbareren Zugriff auf individuelle kriminelle Krisensituationen hat, als die Gerichte, war natürlich an dieser Aufarbeitung intensiv beteiligt. Dies wird von der Öffentlichkeit auch erwartet. Es kann Situationen geben, vor allem im Zusammenhang mit Tötungsdelikten und anderen Gewaltdelikten, in denen gerade auch von den Staatsanwaltschaften eine sachgerechte Reaktion in der Zusammenarbeit mit anderen staatlichen Stellen gefordert ist. Wenn es hier zu Versäumnissen kommt, kann und 
muss dies zu politischen Konsequenzen führen, im Extremfall bis hin zu personellen Konsequenzen. Es muss uns also gelingen, eine Selbstverwaltung der Staatsanwaltschaft zu schaffen, bei der die direkte demokratische Verantwortlichkeit gegenüber dem Parlament in diesem Bereich nicht verloren geht. Für das Fehlen einer solchen Verantwortlichkeit hätte die Öffentlichkeit - meines Erachtens zu Recht - im Krisenfall kein Verständnis.

\section{Erwartung 3: Das Gewicht der Stimme der Dritten Gewalt profitiert von einer Teil- nahme am Haushaltsverfahren}

Der Justiz die Möglichkeit zu geben, ihre Haushaltsmittel eigenverantwortlich einzusetzen, ohne zugleich Ihren Einfluss auf die Beschaffung der erforderlichen Haushaltsmittel zu erhöhen, würde zu einer politischen Asymmetrie führen. Es ist wie beim Wahlrecht: Das Recht zu wählen muss auch einhergehen mit der Möglichkeit gewählt zu werden.

Es vergeht kaum ein Gespräch mit Richterinnen und Richtern, in dem nicht die Geringschätzung der Politik gegenüber den Belangen der Dritten Gewalt beklagt wird. Wer diese Einschätzung etwas gründlicher betrachtet wird schnell feststellen, dass die Tätigkeit der Richterinnen und Richter im politischen Raum von Exekutive und Legislative in Wahrheit ein immens hohes Ansehen genießt. Wie sonst wäre es zu erklären, dass die Politik bei der Steuerung schwieriger Fragestellungen häufig gerade auf richterliche Entscheidungen setzt? Ich nenne nur ein paar Stichworte: Die stetige Ausweitung von Richtervorbehalten bei Grundrechtseingriffen, die Rolle der Gerichte bei der Sterbebegleitung und die jüngst erweiterten Befugnis der Richterinnen und Richter bei der Steuerung auftretender familiärer Probleme im Vorfeld der Sorgerechtsentziehung.

Mithin ist es keineswegs Geringschätzung, die gegenüber der Justiz zum Ausdruck gebracht wird. Es ist der einfache Zusammenhang zwischen Aufgabenbestimmung auf der einen und Mittelzuweisung auf der anderen Seite, der viele Richterinnen und Richter umtreibt. Der Deutsche Richterbund hat die Sorge um die ausreichende Mittelbeschaffung durch den unter Zitat: »Kabinetts- und Parteizwängen« stehenden Justizminister sogar in die Präambel seines Beschlusses zur Selbstverwaltung der Justiz aufgenommen.

Wenn aus einer solchen Stimmung heraus der Wunsch nach mehr Selbständigkeit entsteht, sollten Gesetzgeber und Justizminister nicht weghören. Der Hinweis auf die Haushaltsdisziplin hilft eben nur zum Teil weiter. Die Einhaltung der Haushaltsdisziplin ist erforderlich - auch in der Justiz. Es wäre aus meiner Sicht naiv zu hoffen, dass nach der Implementierung einer Autonomie der Justiz die Haushaltsansätze im kurzund mittelfristigen Bereich stark ansteigen werden. Der Bemerkung Prof. Dr. Classens von der Universität Greifswald in der Zeitschrift Betrifft Justiz ist wenig hinzuzufügen: Alle als legitim anerkannten Bedürfnisse verlangen regelmäßig mehr Geld, als vom Staat insgesamt bereitgestellt wird.

Gleichwohl überwiegen die Chancen, wenn wir die Justiz aus ihrer Abhängigkeit von der Exekutive befreien, wenn es um die Verteilung der Haushaltsmittel geht. 
Ich bin in dieser Frage optimistischer als der Präsident des Bundesverfassungsgerichts Prof. Dr. Papier, der vor dem Hamburgischen Richterverein vor gut vier Jahren ausführte:

»In der Tat dürfte ein unmittelbares Rederecht im Plenum oder im Haushaltsausschuss kaum geeignet sein, den Verlust an Einwirkungs- und Verhandlungsmöglichkeiten zu kompensieren, über die ein durchsetzungskräftiger Justizminister als Regierungs- und in der Regel auch Parlamentsmitglied im Kabinett, in der Fraktion und in den Parteigremien verfügt.«

Die Justiz nimmt in der öffentlichen Wahrnehmung meines Erachtens eine besondere Stellung ein. Wegen der Bedeutung der von ihr zu klärenden Lebenssachverhalte ist sie permanenter Gegenstand der gesellschaftlichen und publizistischen Debatte. Sie hat in ihren verschieden Bereichen tagtäglich Kontakt zu Tausenden von Bürgerinnen und Bürgern. Zugleich genießt sie in Deutschland ein weit höheres Ansehen, als dies etwa Managern, Journalisten oder Politikern zu Teil wird. Sie verdankt dies der sachbezogenen, angemessenen und qualitativ hochwertigen Arbeit, die in der Justiz geleistet wird. Eine Justiz, die den Zusammenhang zwischen den ihr übertragenen Aufgaben und der ihr zugewiesenen Mittel deutlich herausstellt, wäre in einer öffentlichen Debatte aus meiner Sicht authentischer und damit wirkungsvoller als ein Justizminister, der ja nicht kraft eigener Gewalt den Justizgewährleistungsanspruch umsetzt.

Herr Prof. Dr. Papier drückte sodann seine Befürchtung aus, die Justiz könne durch ihre Teilnahme am Haushaltsverfahren ihre »politische Unschuld « verlieren, wenn etwa eine Erhöhung von Richterplanstellen zu Lasten der Planstellen für Lehrer oder Polizisten durchgesetzt werde. Ich halte die Prognose, dass eine selbständige Justiz stärker in macht- und medienpolitische Prozesse miteinbezogen wird, durchaus für zutreffend. Die Justiz wird stärker als bisher öffentlicher Kritik ausgesetzt sein. Daran kann auch das hohe Ansehen der Justiz vermutlich wenig ändern. Es handelt sich um eine zwangsläufige Folge eines gestiegenen Gewichts in der öffentlichen Auseinandersetzung. Diese Einbeziehung ist aber - so verstehe ich die bisherigen Überlegungen aus der Richterschaft - keine »Kehrseite der Medaille«, keine Nebenwirkung, die wir vermeiden könnten. Sie ist ein bewusst und selbstbewusst eingeschlagener Weg.

\section{Erwartung 4: Die rechtspolitische Debatte in und außerhalb der Justiz profitiert von der Belebung durch die Einführung einer Autonomie der Dritten Gewalt}

Lassen Sie mich zum Abschluss die Erwartung ansprechen, die bisher wenig im Fokus steht, aber mir als Justizsenator, der den Grünen angehört, besonders wichtig ist: Ich wünsche eine intensivere Beteiligung der Richterinnen und Richter an der rechtspolitischen Debatte in diesem Land. Diese Beteiligung soll durchaus in den fachlichen Foren auf Tagungen wie dieser und in Zeitschriftenartikeln stattfinden. Sie sollte sich aber nicht hierauf beschränken.

Wir brauchen eine lebendige Diskussion in Deutschland über die Rolle der Gerichte, die auch die Menschen außerhalb der Justiz erreicht. Ich freue mich deshalb über den auf dieser Tagung unternommenen Versuch, auch die mediale Vermittlung des The- 
mas in den Blick zu nehmen. Es genügt nicht, wenn über die Autonomie der Justiz nur in den Richterverbänden und auf den Fluren der Präsidialabteilungen der Gerichte gesprochen wird. Ein Modell der Autonomie der Justiz wird sich nur durchsetzen, wenn dieses in der Vorstellung der Menschen positiv verankert ist. Notwendiger Zwischenschritt hierfür ist, dass es zunächst in der Breite der Richterschaft ankommt. Nur wenn sich die Richterinnen und Richter, die auch in den verschiedensten Bereichen als Multiplikatoren tätig sind, für das Thema begeistern können und es beherzt vertreten, gibt es eine realistische Chance auf eine Einführung der Selbstverwaltung der Justiz.

Meine Erwartung ist: Wenn in der Breite der Richterschaft durch die Diskussion über die Autonomie der Justiz erstmal das Interesse an rechtspolitischen Debatten wieder gestärkt wird, wird sich das auch auf andere Themenkreise auswirken. Eine solche Belebung kann der Qualität und Relevanz der Auseinandersetzung nur gut tun.

\section{Schluss}

Als ich das Tagungsprogramm für unser heutiges Seminar in den Händen hielt, war ich ehrlich erfreut. Die Selbstverwaltung der Justiz ist auf dem besten Wege, von einem akademisch behandelten Thema zu einer konkreten Aufgabenstellung von Justiz, Politik, Wissenschaft und Öffentlichkeit zu werden. Überlegungen ein Seminar dieser Art Ende 2008 in Hamburg zu veranstalten, habe ich nach Empfang des Seminarprogramms zunächst zurückgestellt. Aber »Aufgeschoben ist nicht aufgehoben.« Nicht nur die Politik darf Erwartungen formulieren. Auch Sie werden - zu Recht - Erwartungen an die Politik haben. Dazu gehört vor allem die Frage, wie ein gesetzlicher Rahmen für eine Autonomie der Justiz aussehen kann. Wo sind bundes- und landesgesetzliche Änderungen nötig? Müssen auch Vorschriften aus der Verfassung mit in die Betrachtung einbezogen werden? Brauchen wir ggf. Öffnungsklauseln, die den unterschiedlichen Ausgangssituationen in den Bundesländern Rechnung tragen? Auf einem zweitägigen Seminar werden sich nicht sämtliche dieser Fragen klären lassen. Das Seminar in Hamburg werde ich im Laufe des kommenden Jahres durchführen. Es wird getragen von den Diskussionen, die wir heute und morgen führen - diese gesetzlichen Rahmenbedingungen verstärkt in den Blick nehmen. Meine sehr geehrten Damen und Herren, ich würde mich freuen, vielen von Ihnen bei diesem Diskussionsprozess abermals zu begegnen. 